\title{
Nutrition screening in the Channel Islands: creating a positive nutrition and feeding environment in the Guernsey continuing care sector
}

\author{
L. Harbottle \\ Dept of Nutrition \& Dietetics, Health \& Social Services Dept, Princess Elizabeth Hospital, St Martin's, \\ Guernsey GY4 6UU, UK
}

The Health \& Social Services Department in Guernsey stands outside the jurisdiction of the National Health Service but refers to UK guidelines to inform local practice, including delivery of care to patients within the elderly and elderly mental health long-stay sectors. In 2000 a multidisciplinary nutrition task force implemented a local screening policy, resulting in the introduction of a standardised nutritionscreening tool across all adult wards including the continuing care units. Over the intervening 8 years there have been management and staffing changes and improvements in the physical environment, which together with a focus on nutrition education have resulted in greater staff motivation and better consistency of care and monitoring of nutrition of all patients receiving continuing care in Guernsey.

Audits carried out in 2002 and 2004 and again in 2008, following change to the malnutrition universal screening tool (MUST), demonstrated significant improvements in levels of screening, as a result of the implementation of previous audit recommendations. The recording of height and BMI data has increased from $36 \%$ in 2002 to $80 \%$ in 2004 and $94 \%$ in 2008 ( $n$ 121). At each subsequent audit the numbers of underweight in-patients (BMI $<20 \mathrm{~kg} / \mathrm{m}^{2}$ ) fell from $42 \%$ in 2002 to $24 \%$ in 2004 and $19 \%$ in 2008 .

However, it was observed that other variables of MUST are poorly understood and variably documented. Weight loss and percentage weight loss were only recorded on $58 \%$ and $41 \%$ of records respectively in 2008, while disease-effect score was only documented on $40 \%$ of records. BMI score was often confused with BMI and not completed at all or entered incorrectly.

As a result of the findings of the latest audit the MUST has been further simplified and modified to a form that will be suitable for incorporation within the forthcoming electronic health record system.

The 2007 British Association of Parenteral and Enteral Nutrition screening survey ${ }^{(1)}$ has indicated that nutrition policies frequently do not deliver success at a practical screening level. Key factors contributing to the success of the screening policy and process in Guernsey include strong leadership, managerial support, appropriate equipment (particularly knee-heel callipers and wheelchair scales), ongoing education (especially relating to the ethics and specific aims of nutritional support within this patient group), the central role of the wardlevel nutrition link nurses and a sustained dietetic commitment.

1. Russell C \& Elia M (2007) Nutrition Screening Survey in the UK in 2007. A Report by BAPEN. Maidenhead, Berks.: BAPEN. 